\title{
Universiteit
}

Leiden

The Netherlands

\section{Entre traditions flamande et ibérique. Les oeuvres religieuses flamandes comportant des portraits d'Espagnols (1400-1550)}

Falque, I.M.J.

\section{Citation}

Falque, I. M. J. (2011). Entre traditions flamande et ibérique. Les oeuvres religieuses flamandes comportant des portraits d'Espagnols (1400-1550). Publication Du Centre Européen D'etudes Bourguignonnes, 51, 275-301. Retrieved from https://hdl.handle.net/1887/18523

Version:

Not Applicable (or Unknown)

License: Leiden University Non-exclusive license

Downloaded from: $\quad$ https://hdl.handle.net/1887/18523

Note: To cite this publication please use the final published version (if applicable). 


\title{
INGRID FALQUE
}

\author{
Yniversité de Leyde
}

\section{ENTRE TRADITIONS FLAMANDE ET IBÉRIQUE. LES CEURES RELIGIEUSES FLAMANDES COMPORTANT DES PORTRAITS D'ESPAGNOLS (1400-1550)}

Au sein de l'abondante production picturale des anciens Pays-Bas des XV et XVI' siècles, les tableaux religieux comportant des portraits de contemporains en prière - c'est-à-dire des «portraits dévotionnels $»^{1}-$ ont rencontré un vif succès ${ }^{2}$. De nos jours, on conserve ainsi des centaines d'œuvres comportant un ou plusieurs portrait(s) de dévots, agenouillés en prière devant la Vierge, le Christ, un saint ou encore une scène religieuse narrative. Si la majeure partie des personnes représentées dans ces images sont des Bourguignons, une part non négligeable de ces tableaux ont été commandés par et représentent des étrangers, parmi lesquels un certain nombre d'Espagnols.

Le but de cet article est d'analyser ces œuvres flamandes commandées par ces derniers, afin d'en dégager les particularités. Les commanditaires ibériques ontils préféré un type d'œuvres particulier? Les tableaux flamands comportant des portraits d'Espagnols trahissent-ils une influence des traditions ibériques ? Quelles sont les raisons qui ont poussé ces Espagnols à commander ces œuvres en Flandre?

1 Dans le domaine pictural, la tradition veut que l'on dénomme "portrait de donateur 》 tout portrait d'une personne représentée dans une attitude pieuse. Employée correctement, elle ne peut cependant s'appliquer qu'aux portraits des personnes apparaissant dans des auv res de fondation dont elles sont les commanditaires. Afin d'éviter tout emploi abusif de cette appellation, nous recourons au terme "portrait dévotionnel ", qui recouvre l'ensemble des auvres concernées.

2 Sur le portrait dévotionnel dans les anciens Pays-Bas à la période concernée, nous nous permettons de renvoyer à notre thèse de doctorat, dans laquelle le lecteur trouvera la bibliographie sur le sujet : I. FALQUE, Portrails de dévots, pratiques religieuses et expértence spirituelle dans la peinture des anciens Pays-Bas (1400-1550), thèse de doctorat inédite, Université de Liège, 2009. 
Peut-on distinguer des différences entre les œuvres commandées par des Espaynols établis en Flandre et celles commandées par des Espagnols simplement de passage? Voilà autant de questions auxquelles on s'attachera à apporter des réponses.' Pour ce faire, il importe de bien déterminer les caractéristiques du portrait dévotionnel flamand et celles de la peinture religieuse espagnole de l'époque. Il sera égaienent nécessaire de se pencher sur le phénomène - déjà bien cerné- de l'intérêt porté par les Ibériques à la peinture du Nord des Pyrénées.

\section{Le portrait dévotionnel en Flandre : une présentation}

Le portrait intégré dans l'art religieux existe depuis les premiers siècles du Moyen Âge, mais il connait sans conteste sa période de plein essor à partir de la seconde moitié du XIVE siècle, quel que soit le médium employé ou la région concernée. Sí, jusqu'au XIV siècle, il est avant tout réservé aux souverains, le portrait dévotionnel connaît ensuite une diffusion dans des sphères diverses de la société. Les nobles d'abord, puis les bourgeois et les membres du clergé apparaissent de plus en plus fréquemment en prière dans les œuvres religieuses qu'ils commandent. Ceci est particulièrement vrai dans le domaine de la peinture sur bois flamande du $\mathrm{XV}$ siècle. Par le biais de leur effigie, ces hommes et ces femmes désirent laisser une trace de leur piété et de leur passage sur terre. Oscillant régulièrement entre dévotion sincère et volonté d'exalter leur prestige, les motivations des personnes portraiturées sont bien souvent complexes. Si la fonction première de ces images est avant tout d'assurer le salut de la personne représentée, les effigies peuvent aussi agir sur un plan plus purement spirituel et/ou social, le but étant alors pour le modèle de manifester sa richesse et son statut ${ }^{4}$.

Le corpus des peintures comportant des portraits dévotionnels, produites dans les Pays-Bas entre 1400 et 1550 , est conséquent. À ce jour, nous avons en effet dénombré six cent soixante-trois cuvres, se présentant sous des formats très divers. Il va sans dire que cette variété formelle va de pair avec une diversité de fonctions, d'usages et de significations des images. Les œuvres recensées peuvent être réparties en plusieurs catégories selon les critères suivants : la localisation des portraits dans la structure matérielle de l'œuvre (c'est-à-dire sur les revers ou l'avers des volets,

3 Le présent article apparât ainsi comme un complément au tout récent ouvrage de Didiır Martens consacré à plusieurs types de tetables flamands - les triptyques à volets à doubles compartiments et les retables muraux - destinés à la péninsule ibérique : $D$. MARTENS, $P_{\text {einture }}$ flamande et goût ibérique anx $X V^{e}$ et $X V T^{e}$ siècles, Bruxelles, 2010. Nous tenons à temercier vivement l'auteur pour nous avoir donné l'accès aux épreuves de son ouvrage avant sa parution et pour avoir répondu à nos questions. Nous adressons également nos remerciements à Gilles Docquier pour sa relecture attentive du présent article.

4 La présence de blasons, de motifs héraldiques, de devises et d'autres inscriptions dans des cuures comme le Diptyque de Maarten van Nieuwenhove de Memling (Bruges, Memlingsmuseurn, $n^{\circ}$ inv. O.SJ178.1) doit être replacé darıs ce contexte. Pour une synthèse de ces questions (accompagnée de la bibliographie la plus récente sur le sujet), voir I. Fatous.. Portraits de dévots, op. cit., pp. 13-33. Le lecteur intéressé par les aspects spirituzels et dévotionnels de ces œuvres se référera à la seconde partie de notre thèse (pp. 245-352). 
sur le même panneau que la scène religieuse, dans un diptyque...) ; la présence ou non de saints patrons qui introduisent les dévots; le type de scène religieuse devant laquelle apparaissent les personnes portraiturées (narrative ou hiératique). Bien évidemment, d'autres éléments inhérents aux ceuvres jouent également un rôle prépondérant : la catégorie sociale des dévots portraiturés, leurs attributs, la destination de l'œuvre, la structuration picturale sont autant d'éléments dont il faut tenir compte pour analyser ces images. En envisageant l'ensemble de ces criteres, l'étude du corpus permet de dégager des normes de représentation pour certaines catégories de dévots ou certains types d'cuvres, mais aussi de mettre en évidence des œuvres atypiques ${ }^{5}$.

De manière générale, l'origine sociale et géographique des dévots, ainsi que la catégorie de personnes portraiturées (couples, familles, individu isolé, communautés religieuses ou corporatives) influent sur les modes de représentation des portraits. En effet, certains types de personnes marquent une nette prédilection pour des formules particulières. Ainsi, les personnes représentées seules - et plus particulièrement les hommes laïques - optent le plus souvent pour un portrait intégré sur le même panneau que la scène religieuse ou dans des diptyques dédiés à la Vierge ${ }^{6}$. Au contraire, les familles et les couples affichent une préférence pour les portraits localisés sur la face interne des volets', comme 1'illustre par exemple le Triptyque de la Nativité de Gérard David représentant un couple en prière ${ }^{8}$.

Si le nombre d'œuvres comportant des portraits dévotionnels est très élevé, on ne connaît malheureusement pas l'identité de la grande majorité des dévots représentés. En effet, sur l'ensemble du corpus, $40 \%$ seulement des personnes portraiturées ont pu être identifiées, soit l'équivalent d'un peu plus de deux cent cinquante ceuvres. Parmi elles, si la plupart sont originaires des Pays-Bas, on recense tout de même un certain nombre d'étrangers : de nombreux Italiens parmi lesquels Tommaso Portinari (portraituré à deux reprises par Memling et une fois par Hugo van der (Goes' ${ }^{9}$ ), son concurrent Angelo Tani et son épouse (dans le

5 L'analyse du corpus selon ces critères a été réalisée dans la première partie de notre thèse de doctorat : I. Falque, Portraits de dévots, op. cit., pp. 49-244.

6 On pensera notamment, pour le premier cas, au célèbre Triptyque de Pieter Bladelin de Rogier van der Weyden (Berlin, Gemäldegalerie, $n^{\circ}$ inv. 535) et, pour le second, au Diptyque de Maarten van Nieuwenhove évoqué ci-dessus.

7 Selon toute vraisemblance, des raisons pratiques, liées au nombre souvent élevé de personnes portraiturées et à l'espace dissponible pour placer ces effigies, justifient la prédilection des familles pour les portraits sur les volets, et non sur le même panneau que la scène religieuse.

8 New York, Metropolitan Museum of Art, $\mathrm{n}^{\circ}$ inv. 49.7.20a-b-c. Reproduction dans M. W. AnsworTr, Gerard David. A Purity of Vision in an Age of Transition, New York, 1998, pp. 210-211.

9 Tommaso Portinari et son épouse Maria Baroncelli apparaissent en effet dans le Panorama de la Passion (Turin, Galleria Sabauda, $\mathrm{n}^{\circ}$ inv. 8) et dans un double portrait (New York, Metropolitan Museum of Art, $n^{\circ}$ inv. 14.40.626-627) de Memling, ainsi que dans le célèbre Triptyque Portinari de Hugo van der Goes (Florence, Gallera degli Uffizi, $\mathrm{n}^{\circ}$ inv. 1525). 
Triptyque du Jugement dernier de Memling $\left.{ }^{10}\right)$, le Génois Stefano Raggi portraituré par Joos van Cleve ${ }^{11}$ - mais aussi des Allemands (dont la famille Von Humbracht et la famille colonaise Hackeney, figurée à deux reprises par Joos van Cleve ${ }^{12}$ ). un ou deux Anglais (dont John Donne of Kidwelly et sa famille immortalisés par Yans Memling ${ }^{13}$ ), quelques Portugais (parmi lesquels un membre de la confréric des mariniers de Porto ${ }^{14}$ ), une confrérie estonienne ${ }^{15}$ et, enfin, des Espagnols. Ceuxci constituent la deuxième nation la mieux représentée dans le corpus, après les Italiens. Cela n'est guère étonnant, si l'on tient compte du succès rencontré par l'art flamand đans la péninsule ibérique à cette époque.

\section{L'engouement des Espagnols pour l'art flamand}

L'ars nova flamand a suscité, dès le début du XV siècle, un engouement sans précédent dans l'ensemble de l'Europe occidentale, et plus particulièrement dans le Sud. Dans le domaine de la peinture de chevalet, la Flandre apparait en effet comme l'un des deux pôles artistiques majeurs de l'époque, aux côtés de l'Italie. Rappelons brièvement les différents canaux de diffusion de l'art flamand dans les régions du sud de l'Europe : le développement économique des Pays-Bas bourguignons, l'importance du commerce international - avec comme corollaire l'établissement de marchands et de banquiers des nations étrangères dans les villes flamandes ${ }^{16}$-, les nombreux contacts diplomatiques entre les cours royales et princières, ainsi que la mobilité des artistes ${ }^{17}$ sont autant de voies de diffusion des œuvres flamandes en dehors des territoires bourguignons.

Dans la péninsule ibérique plus précisément, le goût pour la peinture flamande se manifeste déjà à la fin du XIV' siècle, pour s'affirmer ensuite de manière de

10 Dantzig, Museum Narodowe, $n^{\circ}$ inv. SD/4131M.

11 Triptyque de l'Adoration des mages, Gênes, église San Donato.

12 Nicaise Hackeney a commandé à Joos van Cleve deux triptyques identiques représentant la Mort de la Vierge, dans lesquels il apparaît avec son frère et leurs épouses respectives. Le premier triptyque est conservé à Cologne (Wallraf-Richartz Museum, $n^{\circ}$ inv. WRM 430) et le second à Munich (Alte Pinakothek, $\mathrm{n}^{\circ}$ inv. 150-152).

13 Triptyque de la Vierge à l'Enfant (Londres, National Gallery, $\mathrm{n}^{\circ}$ inv. NG 6275).

14 Anonyme des Pays-Bas méridionaux (début du XVIe siècle), Triptyque de la Descente dhe Saint-Esprit (Porto, Igreja de Miragaia).

15 Maitre de la Légende de sainte Lucie, Triptyque de la confrérie des Têtes noires de Tallin (Tallin, Musée des Beaux-Arts).

16 On pensera, par exemple, aux nombreux étrangers établis à Bruges, dont plusieurs sont à l'origine de la commande de célèbres cuvres flamandes. C'est notamment le cas de Tommaso Portinari, le directeur de la filiale brugeoise de la banque des Médicis, évoqué précédemment.

17 Sur ce dernier aspect en particulier, on consultera T.-H. BorcherT, La mobilité des artistes. Aspects du transfert culturel à la veille des Temps modernes, dans Le siècle de Van Eyck. 1430-1530. Le monde méditerranéen et les Primitifs flamands, éd. T.-H. BoRcherT, Gand et Amsterdam, 2002, pp. 32-51. 
plus en plus forte dans le courant du siècle suivant ${ }^{18}$. Les échanges commerciaux expliquent en partie la précocité de cet engouement. Les principaux mécènes des artistes nordiques proviennent en effet des villes ayant des relations commerciales intenses avec les Pays-Bas. Il apparaît, par ailleurs, que les commandes d'cuvres flamandes par des Espagnols se font régulièrement par l'entremise de marchands ibériques installés en Flandre ${ }^{19}$. Il a dû en être notamment ainsi d'un retable de Memling destiné au maître-autel du couvent bénédictin Santa María la Real à Nájera, et dont on ne conserve plus que les trois panneaux du registre supérieur ${ }^{20}$. Les opinions divergent quant au commanditaire de l'œuvre, mais l'on s'accorde généralement pour affirmer que la commande a été passée à Memling vers 1489 par l'entremise de marchands espagnols établis à Bruges ${ }^{21}$.

La vitalité commerciale entre la péninsule et les Pays-Bas n'est cependant pas le seul facteur de l'intérêt espagnol pour l'art flamand. Les nombreux contacts existant entre la cour bourguignonne et les cours d'Aragon et de Navarre, ainsi que le penchant des souverains espagnols pour l'art du nord des Alpes ont également joué un rôle considérable. Selon toute vraisemblance, en 1427, Philippe le Bon invite son peintre de cour et varlet de chambre Jan van Eyck à se joindre à une mission diplomatique au royaume d'Aragon destinée à arranger le mariage avorté du duc avec la nièce d'Alphonse V, Isabelle d'Urgel. Le peintre aurait été chargé

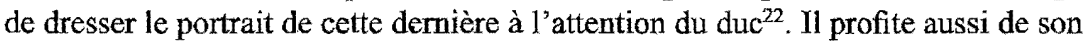
séjour pour visiter Valence et Barcelone, qui sont alors deux centres artistiques importants. Il est plus que probable qu'il y a rencontré des artistes locaux. En 1428-1429, le peintre fait à nouveau le voyage vers la péninsule - cette fois au scin d'une délégation se rendant au Portugal, en vue du mariage entre le duc et Isabelle de Portugal. Il semble qu'à cette occasion, le peintre se rend à la cour de Juan 11 de Castille à Valladolid ${ }^{23}$. On remarquera que si le souverain castillan n'a pas possédé

$18 \mathrm{~J}$. K. STEPP, Mécénat espagnol et art flamand au XVT siècle, dans Splendeurs d'Espagne et les villes belges. 1500-1700, vol. l, éd. J.-M. Duvosquec et I. VANDevivere, Bruxelles, 1985, p. 248.

19 Ibidem.

20 Les trois panneaux en question sont conservés au Koninklijk Museum voor Schone Kunsten d'Anvers $\left(\mathrm{n}^{\circ}\right.$ inv. 778-780). Selon toute vraisemblance, le thème principal du retable était l'Assomption de la Vierge. Voir en demier lieu D. Martens, Peinture flamande et goût ibérique, op. cit., pp. 100-107.

21. Selon Dirk De Vos, le retable est une commande de la famille Nảjera - dont le nom est fréquernment associé au marché de la laine espagnole à Bruges à cette époque. La commande aurait été passée par l'um de ses membres vivant en Flandre. Cf. D. DE vos, Hans Memling, op. cit., p. 289. Selon Till-Holger Borchert, qui se base sur le contexte historique de l'abbaye à l'époque de la commande, celle-ci a dû être passée par l'abbé lui-même. Cf. Bruges ef la Renaissance. De Memling à Pourbus. Notices, Bruges, 1998, pp. 21-22. Didier Martens opte lui aussi pour cette seconde hypothèse.

22 Sur le voyage en Aragon et le présumé portrait, voir C. STERLNa, Jan van Eyck avant 1432, dans Revue de l'art, 33, 1976, pp. 32-33 et C. Penám y PeMArtín, Juan van Eyck y España, Cádiz, 1969, pp. 29-34.

23 C. SterLING, Jan van Eyck avant 1432, op. cit., pp. 33-35. 
d'œuvre eyckienne, malgré le passage du peintre à sa cour, il porte cependant un certain intérêtà la peinture flamande. En atteste la présence du Triptyque de la Vierge (dit Triptyque de Miraftores) de Rogier van der Weyden dans ses collections ${ }^{27}$, En 1445, cette œuvre est léguée par Jean II au couvent de Miraflores à Burgos. où il souhaite être inhumé. Son fils Henri $\mathrm{IV}$ a, pour sa part, fait don au couvent d'El Parral à Ségovie d'une cuvre eyckienne représentant «l'histoire de la dédicace de l'Église », comme le stipule le cartulaire du monastère ${ }^{25}$. On s'accorde aujourd 'hui pour considérer la Fontaine de vie eyckienne conservée au Prado comme l' rewure mentionnée dans le cartulaire ou comme une copie de celle-ci ${ }^{26}$.

Le goût manifeste d'Alphonse $V$ pour la peinture flamande semble également avoir été un déclencheur non négligeable en Aragon. Le souverain apparaîi en effet comme un collectionneur et un mécène important pour les artistes flamands. Il posséde non seulement quelques cuvres de Rogier van der Weyden et de Jan van Eyck (notamment un Saint Georges aujourd'hui disparu ${ }^{27}$ ), mais aussi de nombreuses tapisseries ${ }^{28}$. Son goût pour la tapisserie flamande est en outre attesté par la présence d'un licier flamand à son service, un certain Guillaume d'Ixelles. En 1431, Alphonse V envoie ce dernier et son peintre de cour Luís Dalmau aux Pays-Bas. On avance parfois que les deux artistes ont alors pour mission d'étudier le savoir-faire local en matière de tapisseries et d'engager de la main-d ceuvre en vue de la fondation d'une manufacture en Espagne ${ }^{29}$. Dalmau séjourne en terres bourguignonnes de 1431 à 1436 et profite de sa présence sur place pour se familiariser avec la technique picturale flamande et étudier plusieurs œeuvres, dont le célèbre Polyptyque de l'Agneau mystique des frères Van Eyck (cf. infra).

Dans un souci probable d'émulation de leurs souverains et parce que l'art flamand jouit dès le $X V^{\vee}$ siècle d'une renommée internationale, bon nombre de nobles espagnols commandent, à leur tour, des peintures flamandes, comme nous le verrons plus loin. Outre ces cuvres de commande, on assiste également, dans le courant du XVE siècle, à un développement tout à fait conséquent du marché de l'art flamand dans la péninsule ibérique. Des marchands et des artistes espagnols établis en Flandre se spécialisent dans l'exportation de tableaux de dévotion en

24 Berlin, Gemäldegalerie, $n^{\circ}$ inv. 534A.

25 Le cartulaire est conservé à la Bibliothèque nationale de Madrid, section des manuscrits, $n^{\circ} 19412$. La mention apparaît au $f^{\circ} 54:$ : $E$ otrossi dio el dicho señor rey un retable rico de pinsel de Flandes que tiene la ystoria de la dedicación de la iglesia ".

26 Madrid, Museo del Prado, $n^{\circ}$ inv. 1.511. Sur cette cuvre problématique, son attribution et sa datation, nous renvoyons à E. Bermeso, La Pintura de los primitivos flamencos en España, vol. 1, Madrid, 1980, pp. 47-50.

27 C. Penám y Pemartin, Juan van Eyck y España, op. cit, pp. 37-39 et J. Yarza Luaces, La couronne d'Aragon et la Flandre, dans Le siècle de Van Eyck. 1430-1530, op. cit., p. 129.

28 J. YARZA LuACES, La couronne d'Aragon et la Flandre, op. cit., p. 129.

29 A. Simonson Fucas, The Virgin of the Councillors by Luis Dalmau (1443-1554). The Coniract and its Eyckian Execution, dans Gazette des Beaux-Arts, 99, 1982, p. 53, n. 8 et T.-H. BORCHERT, La mobilité des artistes, op. cit., p. 43. 
Espagne. La plupart de ces cuvres sont écoulées sur le marché libre, lors des foires annuelles, dont la plus célèbre est sans conteste celle de Međina del Campo ${ }^{30}$. Cette foire, dont la durée s'élève alors à cent jours répartis en deux sessions entre mai et octobre, accueille des marchands de nombreux pays et les objets d'art flamands y sont vendus en très grande quantité aux membres de la cour castillane, aux nobles et aux bourgeois. La vigueur de l'exportation de l'art flamand dans la péninsule explique le nombre important d'œuvres flamandes encore conservées aujourd'bui dans les musées et églises espagnols.

Les causes de cet intérêt grandissant des Espagnols - tant artistes que commanditaires - pour la peinture flamande sont également bien connues : la technique de la peinture à l'huile, jusqu'alors inégalée, et le réalisme symbolique des images produites par les " Primitifs flamands 》 apparaissent en effet comme particulièrement novateurs dans la péninsule. À cette époque, le gothique international domine encore très largement la peinture espagnole : les peintres castillans et aragonais peignent à la détrempe, recourent très largement aux fonds d'or et possèdent une moins bonne maitrise du rendu des corps et de l'espace ${ }^{31}$. Au cours du XV siècle, la diffusion des œuvres flamandes dans la péninsule ibérique amène les peintres espagnols à se familiariser avec l'ars nova du Nord, sans compter que de nombreux artistes flamands s'installent en Espagne, diffusant ainsi leur savoir-faire et leurs connaissances techniques ${ }^{32}$. Par ailleurs, il n'est pas rare que des peintres ibériques suivent une formation aux Pays-Bas, si bien qu'au fil du temps, les différences stylistiques et techniques s'amenuisent ${ }^{33}$.

30 Sur le marché de l'art en Espagne au XV siècle, on consultera notamment M.-T. AlVArEZ, Artistic Enterprise and Spanish Patronage: The Art Market during the Reign of Isabel of Castile (1474-1504), dans Art Markets in Europe, 1400-1800, éd. M. NorTt et D. Ormron, Aldershot, pp. 45-59.

31 D. Martens, Peinture flamande et goût ibérique, op. cif., p. 12. Sur la peinture espagnole des $\mathrm{XV}^{e}$ et XVI $\mathrm{XI}^{\mathrm{e}}$ siècles, on consultera notamment P. Silva Maroto, Pintura hispanoflamenca castellana: Burgos y Palencia. Obras en tabla y sarga, Valladolid, 1990 ; P. Silv MAroto, Pintura hispanoflamenca, Madrid, 1993 ; Catalogue de l'exposition La Clave Flamenca en los primitivos Valencianos, éd. F. Benito Domínech et J. Gómez Frechina, Valence, 2001 ; P. Silva Maroto, El arte en España en la época del primer Marqués de Santillana (13981458), dans El Marqués de Santillana. 1398-1458. Los albores de la España Moderna. El Humanista, éd. J. YarZA LuACEs et al., Valence, 2001, pp. 155-191.

32. On pensera notamment à Louis Allyncbrood, un peintre brugeois qui s'installe en 1439 à Valence.

33 Il est d'ailleurs révélateur de constater que l'on parle généralement d'art « hispano-flamand » pour caractériser la peinture espagnole de cette époque. Pilar Silva Maroto précise toutefois que, bien qu'elle soit entrée dans le vocabulaire courant des auteurs travaillant sur la peinture espagnole du $X V^{*}$ siècle, cette expression ne se justifie pas réellement puisque les artistes espagnols n'ont pas « copié littéralement les modèles du Nord, ils les ont transformés pour les faire correspondre aux goûts et souhaits de leurs commanditaires, ainsi qu'aux exigences et caractéristiques du marché artistique local $»$. Cf. P. Siva Maroto, La couronne de Castille et la Flandre, op. cit., p. 143. 
Enfin, il importe aussi de signaler que les Primitifs flamands ont rapidenent suscité l'admiration des étrangers pour l'innovation dont ils ont fait preuve dans le domaine de l'art du portrait. Dès les années 1420-1430, alors que les artustes du reste de l'Europe perpétuent la tradition du portrait de profil du siècle précédent (largement influencée par l'Antiquité classique en Italie), les peintres des PaysBas développent un art du portrait extrêmement novateur ${ }^{34}$. Des artistes comme le Maître de Flémalle et Jan van Eyck parviennent à capter la présencé. la psychologie et la réalité de leurs modèles par le recours à la formule du portrait de trois-quarts et grâce à leur technique picturale permettant un rendu extrêmement réaliste des textures. De ce fait, il n'est pas étonnant que de nombreux étrangers désirant acquérir une peinture flamande aient souvent opté pour un portrait .. soit indépendant, soit intégré dans une ceuvre religieuse. Dans ce dernier cas, il peut alors s'agir d'une ouvre de dévotion privée ou, plus fréquemment, d'un retable destiné à leur chapelle privée.

$\mathrm{Si}$, parmi les étrangers ayant fait appel à des peintres flamands pour réaliser leur portrait dans une cuvre religieuse, les Italiens sont les plus nombreux, les Espagnols ne sont pas en reste. En effet, nous avons pu recenser treize auves comportant des portraits dévotionnels commandées par, ou représentant des Espagnols. À ce total, il faut encore ajouter neuf autres tableaux dont la provenance ancienne suggère très fortement que les personnes représentées sont originaires de la péninsule ibérique, sans que l'on puisse les identifier plus précisément.

\section{Le portrait dévotionnel : Flandre vs péminsule ibérique}

En 1443, soit un peu moins d'une dizaine d'années après le retour de Luís Dalmau en Espagne suite à sa mission en Flandre, les membres du Conseil des Cent de Barcelone commandent au peintre aragonais un retable qu'ils destinent à leur chapelle privée à l'hôtel de ville (fig. 1). Connu aujourd'hui sous le nom La Vierge des conseillers, ce retable se présentait à l'origine sous la forme d'un panneau ogival doté d'une prédelle, aujourd'hui perdue. Fait exceptionnel, le contrat passé entre Dalmau et les membres du conseil a été conservé et permet de constater que l'artiste a respecté les volontés de ses clients ${ }^{35}$. En effet, comme le stipule le document, le retable représente la Vierge à l'Enfant avec saint Jean,

34 Il suffit, pour s'en convaincre, de comparer deux cuvres plus ou moins contemporaines, comme le Double portrait d'un couple du Maître de Flémalle (Londres, National Gallery, $\mathbf{n}^{\circ}$ inv. NG 653.1-2, vers 1435) et le Portrait de Leonello d'Este de Pisanello Bergame, Accademia Carrara, $\mathrm{n}^{\circ}$ inv. 919, vers 1440). Pour une bistoire du portrait à la Renaissance, le lecteur consultera L. CAMPBEL, Renaissance Portraits. European Portrait Painting in the 14th, 15th and 16th Century, Londres et New Haven, 1990.

35 Le contrat a été publié en anglais dans A. Simonson Fucrs, The Virgin of the Councillors, op. cit., pp. 46-48 et dans C. M. Richardson, K. WoOds et M. W. FrankLIN, Renaissance Art Reconsidered: An Anihology of Primary Sources, Malden, Oxford et Carlton, 2007, pp. 163-186. 


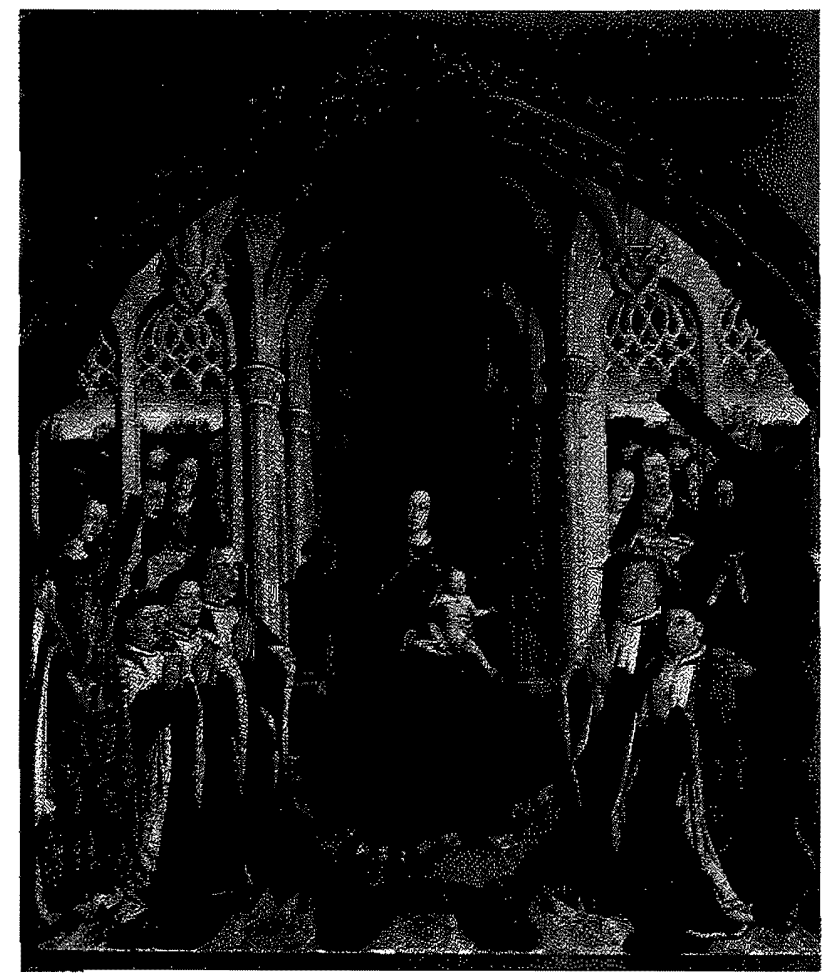

Figure 1

sainte Marie-Madeleine et les armes de la ville de Barcelone. Plus intéressant pour notre propos, le contrat précise que le panneau et le cadre devaient être réalisés « en * bon bois flamand ». Cette spécification prouve, si besoin était, l'attention portée à la technique flamande en terre espagnole. Dans le cas précis de la Vierge des conseillers, l'intérêt de Dalmau pour la peinture des Pays-Bas va beaucoup plus loin : les anges situés de part et d'autre de la Vierge sont une référence explicite aux anges musiciens du Polyptyque de l'Agneau mystique des frères Van Eyck et prouvent par là même que Dalmau a mis son séjour flamand à profit pour étudier l'art des grands peintres flamands de l'époque ${ }^{36}$. L'impact de l'ars nova flamand sur la Vierge des conseillers ne se limite par ailleurs pas à cet emprunt : comme l'a remarqué Anne Simonson Fuchs, la formule du retable composé d'un panneau simple et d'une prédelle correspond davantage aux standards nordiques qu'aux traditions catalanes ${ }^{37}$. Enfin, la manière dont Dalmau s'est attaché à représenter les

36 On remarquera avec intérêt que le contrat stipułe que les personnages devaient se détacher d'un fond d'or et non pas apparaître dans un intérieur réaliste, avec les anges musiciens et le paysage à l'arrière-plan.

37 A. Stmonson Fuchs, The Virgin of the Councillors, op. cit., p. 48. 
cinq dévots - répartis de part et d'autre de la Vierge à la même échelle qu'elle et en compagnie de leurs saints patrons Eulalie et André - trahit également une influence précoce de Jạn van Eyck et, plus généralement, des modes de représentation du portrait dévotionnel dans la peinture flamande contemporaine.

Pourtant, de manière plus générale, les tableaux espagnols de la première moitié du XV siècle comportant des portraits dévotionnels sont encore très largement tributaires de la tradition gothique, selon laquelle les dévots apparaissent à échelle réduite et sans réelle relation avec les personnages saints, objets de leur dévotion. Représentés de la sorte, ils ne sont jamais vraiment intégrés dans la scène religieuse. Cette tendance se perpétue d'ailleurs bien au-delà de 1450 , comme l'atteste notamment la Pietà avec un couple en prière de Fernando Gallego datée des environs de $1470^{38}$. Au contraire, les peintres flamands se sont rapidement attachés à créer des liens entre les saints et les dévots portraiturés, notamment en les représentant à la même échelle, en les intégrant dans le même espace pictural ou encore en créant des liens visuels - voire physiques - entre eux.

Deux remarques s'imposent: premièrement, au fur et à mesure que l'on avance dans le temps - et donc que l'art flamand se diffuse dans la péninsule ibérique - les peintres espagnols intègrent en quelque sorte davantage les leçons de leurs homologues septentrionaux, comme l'avait fait Luís Dalmau à une date relativement précoce. Ensuite, les ceuvres espagnoles dans lesquelles les portraits sontreprésentés de manière novatrice sont bien souvent dues à des artistes originaires de ou formés dans ces régions. Cela est notamment le cas du Retable des arges, appelé aussi Retable du marquis de Santillana du nom de son commanditaire le marquis Don Iñigo Lopez de Mendoza ${ }^{39}$. Il s'agit d'une cuvre de Jorge Ingles, un peintre vraisemblablement d'origine anglaise et formé en Flandre avant de passer sa carrière en Castille. Comme nous le renseigne le codicille du testament du marquis, daté de 1455, le retable était destiné à la chapelle de l'hôpital de Buitrago qu'il avait fondé quelque temps auparavant ${ }^{40}$. Il s'agit d'un retable mural divisé en travées verticales et doté d'une prédelle représentant des personnages en buste (ici, les Pères de l'Église). La travée centrale comporte une statue de la Vierge à l'Enfant, tandis que les travées latérales représentent les commanditaires de l'œuvre en prière.

38 Madrid, Museo del Prado, $\mathrm{n}^{\circ}$ inv. P02998. Reproduction dans Le siècle de Van Eyck. 14301530, op. cit., p. 148.

$39^{\circ}$ Guadalajara, Museo del Palacio de los Mendoza. Reproduction dans le catalogue de l'exposition El Marqués de Santillana. 1398-1458, op. cit., p. 81.

40 Cf. P. Silva Maroto, El arte en España, op. cit., pp. 181-182, ainsi que plus récemment L. M. F. Bosch, Art, Liturgy and Legend in Renaissance Toledo. The Mendoza and the Iglesia Primada, University Park, 2000, pp. 86-87. Le testament de Don Iñigo Lopez de Mendoza a été publié par F. LAYNA Serrano, Historia de Guadalajara y sus Mendoza en los siglos XVY $X V$, t. 1, Madrid, 1942, pp. 325-333. 
D'un point de vue formel et structurel, le Retable des anges respecte donc la typologie des retables ibériques ${ }^{41}$. II comporte cependant une particularité, à savoir la place accordée aux portraits de dévots et la manière dont ils sontreprésentés. Ceuxci occupent en effet les deux panneaux situés de part et d'autre de l'effigie sculptée de la Vierge. Don Iñigo Lopez de Mendoza apparait à gauche -- donc à dextre. la position d'honneur réservée à l'homme - et son épouse Catalina Súarez de Figucuroa à droite. Situés dans une pièce ouverte sur un paysage, les époux sont tous deux agenouillés les mains jointes sur un prie-Dieu disposé devant un drap d'honneur. Les détails accordés à la représentation de l'espace. ainsi que l'intégration des dévots dans ce lieu trahissent une nette influence du portrait dévotionnel flamand. On a notamment rapproché ces effigies de celles de Nicolas Rolin et de Guigone de Salins dans le célèbre Retable du Jugement dernier que Rogier van der Weyden a réalisé vers 1443-1450 pour la chapelle de l'Hôtel-Dieu de Beaune. Le chancહliè de Bourgogne et son épouse y sont en effet représentés sur deux panneaux distincts, de part et d'autre de statues en grisaille des saints Antoine et Sébastien. Ils sont par ailleurs agenouillés sur un prie-Dieu devant un drap d'honneur. Cette comparáson fait toutefois abstraction d'un élément compositionnel important des portraits de Jorge Inglés : les dévots sont portraiturés dans un environnement réaliste, à savoir une pièce évoquant un intérieur domestique ouvert sur l'extérieur. Or, il $s$ 'agit là d'un trait caractéristique de nombreux portraits dévotionnels flamands de l'époque. En effet, outre le fait que les peintres flamands se plaisent à situer leurs personnages dans leur environnement quotidien (un intérieur domestique ou ecclésiastique), il est extrêmement fréquent qu'une ouverture sur un paysage soit ménagée à l'arrière-plan, suggérant ainsi l'endroit par lequel ils sont passés pour pénétrer dans ce lieu de prière. Ce phénomène s'observe notamment dans de nombreux tableaux d'Hans Memling, comme sa Vierge à l'Enfant avec un homme en prière et saint Antoine ${ }^{42}$ ou encore son Diptyque de Maarten van Nieuwenhove. Par son mode de représentation des portraits, le Retable des anges de l'hôpital de Buitrago montre ainsi un autre aspect de l'influence de la peinture flamande sur la . peintre espagnole : la représentation des dévots.

Le Retable de Sopetrán - dont on ne conserve plus que quatre panneaux représentant l'Annonciation, la Nativité, la Dormition de la Vierge et un jeune homme en prière dans un intérieur d'église - est un autre bel exemple de cette influence flamande sur l'art du portrait dévotionnel ibérique (fig. 2). Daté des années 1460-1470, ce retable provient du monastère de Nuestra Señora de Sopetrán, dans lequel le marquis de Santillana s'est beaucoup investi, tant d'un

41 Sur les retables ibériques, nous renvoyons à J. Berg Sobré, Behind the Altar Table. The Development of the Painted Retable in Spain, 1350-1500, Columbia, 1989, ainsi qu'au catalogue de l'exposition Polyptyques. Le tableau multiple du Moyen Age au vingtième siècle, Paris, 1990, pp. 41-42 et à C. Limentani Virbis et M. Pretrogrovanna, Retables. L'âge gothique et la Renaissance, Paris, 2001, pp. 9-35.

42 Ottawa, National Gallery of Canada, $\mathrm{n}^{\circ}$ inv. 6191. Reproduction dans B. G. LANE, Hans Memling. Master Painter in Fifteenth Century Bruges, Londres et Turnhout, 2009, fig. 67. 


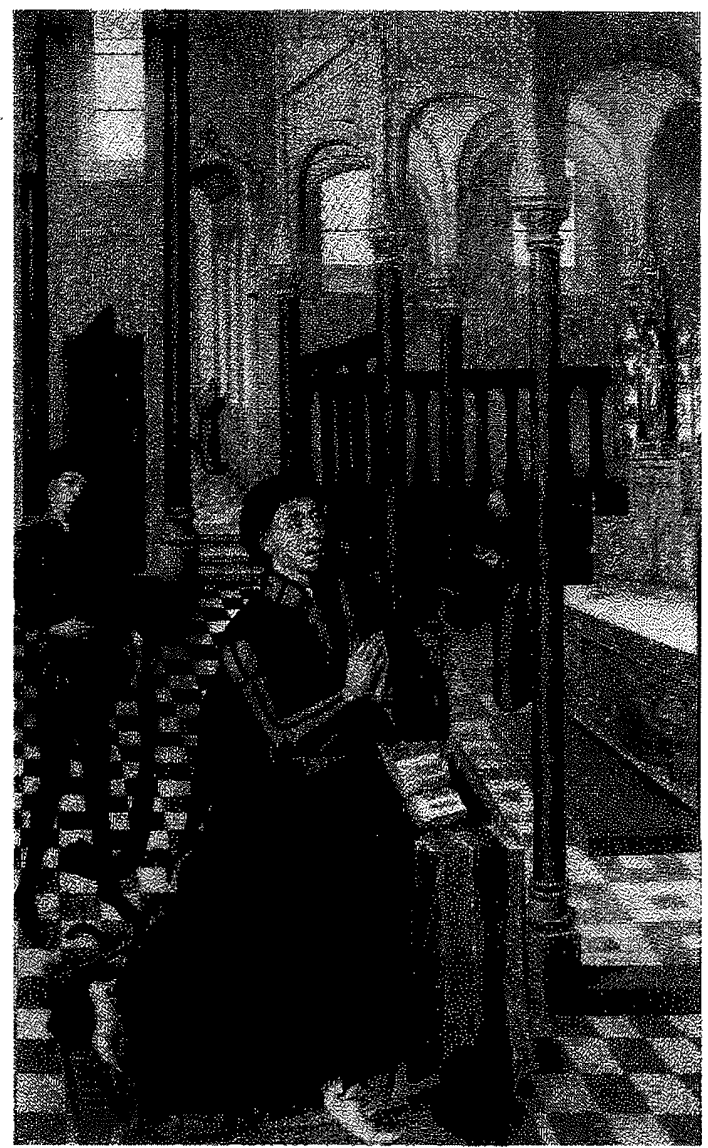

Figure 2

point de vue juridique qu'artistique ${ }^{43}$. L'influence des Primitifs flamands se manifeste non seulement dans le style général des panneaux et dans la composition de l'Annonciation, mais aussi plus particulièrement dans la représentation du dévot en prière. On peut en effet le comparer, entre autres, au volet gauche du Retable de l'Assomption de la Vierge commandé vers 1450 par Philippe de Ternant et son

$43^{~ A ̀ ̀ ~ c e ~ s u j e t, ~ v o i r ~ n o t a m m e n t ~ L . ~ M . ~ F . ~ B o s c h, ~ A r t, ~ L i t u r g y ~ a n d ~ L e g e n d ~ i n ~ R e n a i s s a n c e ~ T o l e d o, ~}$ op. cit., pp. 103-106. Bien que les armes représentées sur le drap qui recouvre le prie-Dieu du dévot portraituré sur le quatrième pannean soient celles des Mendoza, l'identité du dévot est encore sujette à caution. À ce jour, deux hypothèses sont retenues : il pourrait s'agir soit de Diego Hurtado de Mendoza, le fils aîné du marquis de Santillana, soit d'une commémoration posthume du marquis. Dans ce cas, la commande aurait pu être passée par son fils le cardinal Pedro Gonzáles de Mendoza. 
épouse pour la chapelle de leur château à Ternant ${ }^{44}$. Dans les deux cas, le dévot est agenouillé sur un prie-Dieu, sur lequel se trouve un livre de prière ouvert et ce, devant un autel sur lequel est posé un retable.

Bien que la peinture hispano-flamande des XVet $X V^{*}$ siècles ne soit pas le sujet principal de cet article, il est intéressant de constater qu'au fil du temps, les peintres castillans et aragonais intègrent davantage et surtout de mieux en mieux les modalités de représentation du portrait dévotionnel flamand : les effigies sont de plus en plus réalistes et toujours mieux insérées dans la scène religieuse. Réalisé vers 1485 par Bartolomé Bermejo et Rodrigo de Osona, le Triptyque de la Vierge de Montserrat en est d'ailleurs un exemple particulièrement éclairant ${ }^{45}$.

\section{Les portraits dévotionnels flamands commandés par des Espagnols}

À notre connaissance, pour la période concernée ici, toutes les ouvres flamandes comportant des portraits dévotionnels d'Espagnols identifiés que l'on conserve datent des dernières décennies du XVe siècle ou de la première moití du XVY siècle. La plupart d'entre elles sont des commandes émanant de la noblesse et de prélats haut placés. On recense bien l'un ou l'autre riche bourgeois, mais ceux-ci sont très nettement minoritaires. Cette répartition sociale contraste assez fortement avec celle des commanditaires flamands de portraits dévotionnels, pour la plupart issus de la haute bourgeoisie urbaine. En effet, grâce aux travaux de Jean C. Wilson et Marina Belozerskaya, il est bien établi qu'aux Pays-Bas, les clients privilégiés des artistes peintres sont les riches bourgeois. Par souci d'émulation du duc, la noblesse bourguignonne préfère nettement centrer son mécénat sur des cuvres $d^{2}$ art plus luxueuses, comme les manuscrits enluminés et les objets orfévrés. Réalisés avec des matériaux plus précieux que le bois et les pigments colorés employés pour la peinture de chevalet, ces objets ont plus de valeur et mettent mieux en exergue le statut et la richesse de leurs commanditaires ${ }^{46}$. Cette différence entre les commanditaires flamands et espagnols s'explique sans conteste par le prestige dont jouit la peinture flamande, ainsi que par la valeur qu'on lui accorde dans la péninsule ibérique, comme nous l'avons vu précédemment. Pour un membre de la noblesse castillane ou aragonaise, revenir des Pays-Bas avec une cuvre flamande comportant son effigie devait être tout aussi prestigieux que la

44 Sur cette cuvre, on consultera R. JouRnet, Deux retables du XVie siècle à Ternant (Nièvre), dans Annales littéraires de l'Université de Besançon, t. 49, 1963, pp. 5-45 et M. GAUTHIER, Ternant (Nière). Son histoire et ses triptyques, Bourbon-Lancy, 1968.

45 Acqui Terme, cathédrale. Reproduction dans Le siècle de Van Eyck. 1430-1530, op. cit, p. 136.

46 Voir à ce sujet J. C. Wusson, Painting in Bruges in the Close of the Middle Ages. Studies in Society and Visual Culture, University Park, 1998 ; M. BELOZERSKAYA, Rethinking the Renaissance: Burgundian Art Accross Europe, Cambridge et New York, $2002 ; \mathrm{H}$. Wrssman, Luxury Bound. Illustrated Manuscript Production and Noble and Princely Book Ownership in the Burgundian Netherlands (1400-1550), Turnhout, 2010. 
possession d'une pièce orfévrée, puisqu'ils imitent par là même leur souverain. Il importe par ailleurs d'établir une distinction entre les Espagnols établis dans les anciens Pays-Bas et ceux de passage dans la région. Parmi les treize dévots portraiturés identifiés avec certitude, seuls dels sont des Espagnols installés en Flandre. Il s'agit de Jean de Sedano, à quî l'on doit la commande à Gérard David de deux œuvres religieuses comportant son effigie, et de Diego de Guevara dont il sera question plus loin.

Jean de Sedano est un marchand originaire de Santa Maria del Campo, un petit village des environs de Burgos. Arrivé à Bruges à une date indéterminé, il y est reconnu bourgeois et y épouse en 1486 Marie Caignet, la fille d'un négociant brugeois. La première des deux cuvres que Jean de Sedano et son épouse ont commandées à David est le Triptyque de la Vierge à l'Enfant dit Triptyque Sedano, que l'on date des environs de 1490 (fig. 3) ${ }^{47}$. Jean de Sedano et son fils y apparaissent sur le volet gauche, tandis que Marie Caignet figure sur le volet droit. Le parneau central est occupé par une Vierge à l'Enfant trổnant dans un paysage nordiqué. Le Triptyque Sedano apparaît ainsi comme étant une cuvre typiquement flantande, commandée par un Espagnol vivant aux Pays-Bas et habitué aux traditions artistiques locales, tant du point de vue de l'iconographie (une Vierge à l'Enfant dans un paysage) que du format (le triptyque) et des modes de représentation des portraits sur les volets de l'cuvre. En effet, d'après les auvres conservées, les couples et les familles ont marqué une très nette prédilection pour les triptyques comportant leurs effigies sur la face intérieure des volets ${ }^{48}$. En outre, on constatera avec intérêt que l'cuvre comporte une référence à l'un des plus grands chefsd'œuvre de la peinture brugeoise, à savoir la Vierge au chanoine Van der Pale de Jan van Eyck ${ }^{49}$. Le groupe central de la Vierge a l'Enfant de David est en effet très largement inspiré de la Vierge à l'Enfant eyckienne. Comme l'a proposé Maryan W. Ainsworth, cet emprunt revêt une signification spécifique, tant pour le peintre que pour le commanditaire : pour Gérard David, il s'agit d'un moyen d'apparaître comme le digne successeur de Van Eyck à Bruges, tandis qu'il permet à Jean de Sedano d'exprimer son prestige en imitant les membres de la cour castillane qui possèdent des cuvres flamandes ${ }^{50}$.

47 La destination originale du Triptyque Sedano n'est pas connue, mais les dimensions moyennes du triptyque plaident en faveur d'une cuvre destinée à un autel domestique ou à une petite chapelle privée. En 1890, l'œuvre se trouve dans une collection privée espagnole, ce qui suggère que l'œuvre fut envoyée à une date inconnue en Espagne (peut-être par des descendants de Jean de Sedano). Voir H. Adyrémar, Le Musée national du Louvre. I. Les Primitifs flamands. I. Corpus de la peinture des anciens Pays-Bas méridionaux au XVE siècle, Bruxelles, 1962, $\mathrm{n}^{\circ} 89$.

48 Cf. I. Falque, Portraits de dévots, op. cit., pp. 92-106.

49 Bruges, Groeningemuseum, $\mathrm{n}^{\circ}$ inv. 0.161.I.

50 M. W. Ainsworth, Gerard David. A Purity of Vision in an Age of Transition, op. cit., pp. 164-165. 


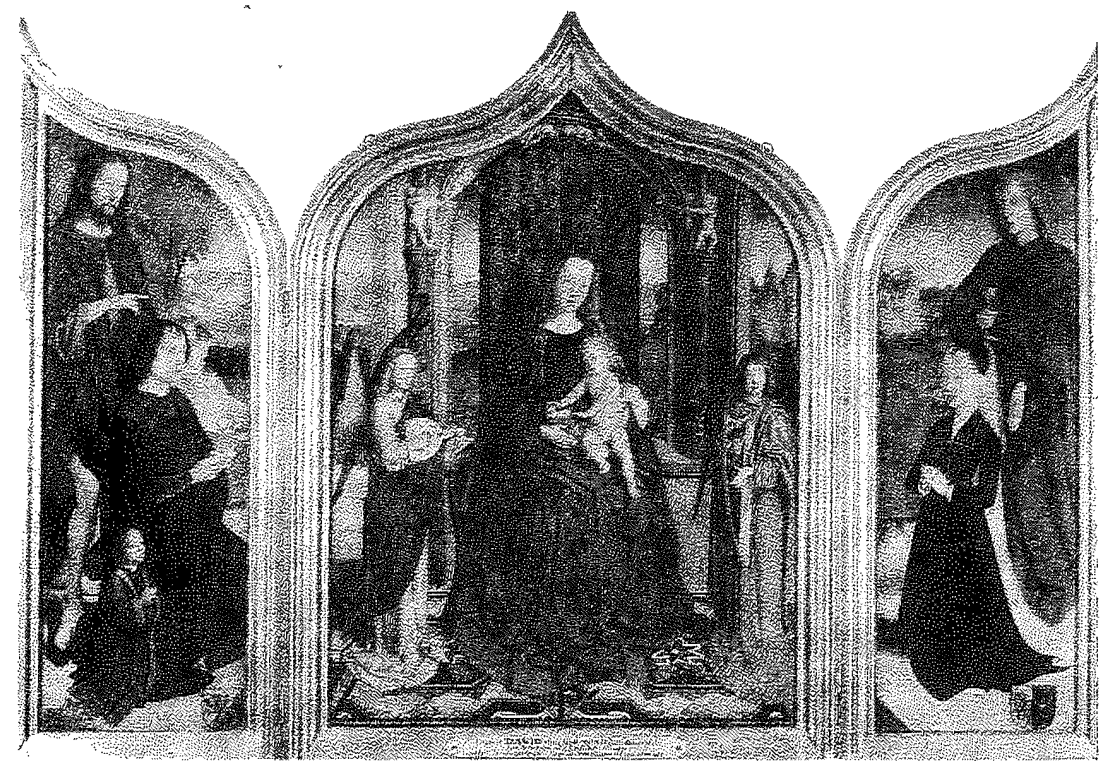

Figure 3

Quelques années après la commande de ce triptyque, Jean de Sedano a fait à nouveau appel à Gérard David, cette fois pour la réalisation d'un tableau destiné à la chapelle du Saint-Sang de Bruges ${ }^{51}$. Cette ceuvre représente les Noces de Cana, . auxquelles assistent les dévots. Vêtu du tabart de la confrérie du Saint-Sang dont il est membre depuis 1501, Jean de Sedano apparaît avec son fils dans le coin inférieur gauche du panneau, tandis que son épouse figure dans le coin inférieur droit. Du point de vue de l'intégration des portraits, il s'agit là aussi d'une ceuvre typiquement flamande, ne répondant nullement aux critères espagnols : les portraits sont représentés à même échelle que les personnages saints et parfaitement intégrés dans la scène religieuse. Ici, la destination de l'ouvre - la chapelle de la confrérie du Saint-Sang sur la place du Franc à Bruges - justifie le caractère pleinement flamand du retable. Elle explique en outre le thème de l'œuvre, que l'on rencontre peu dans la peinture flamande des $\mathrm{XV}^{\mathrm{e}}$ et $\mathrm{XVI}^{\mathrm{e}}$ siècles. Les Noces de Cana sont en effet une préfiguration de l'Eucharistie.

51 Paris, Musée du Louvre, $\mathrm{n}^{\circ}$ inv. 1995. Reproduction dans H. VAN Miegroet, Gérard David, Anvers, 1989 , p. 308 , 
Le Triptyque Sedano et les Noces de Cana sont deux cuvres commandées par un Espagnol établi en Flandre et bien ancré dans la vie locale brugeoise. En effet, l'intégration de Sedano se manifeste non seulement par son mariage el son appartenance à l'une des plus prestigieuses confréries de la ville, mais aussi par le style des ceuvres qu'il a commandées. Toutes deux attestent qu'il était bien accoutumé aux traditions artistiques locales. Il en va de même pour don Diego de Guevara, dont il sera question plus loin, et qui a passé une grande partie de sa vie en terre flamande. Par contre, plusieurs Espagnols de passage aux Pays-Bas et désirant rapporter chez eux une ceuvre représentative du savoir-faire flamand n' ont pas totalement franchi le cap ; ce sont alors les peintres qui se sont partiellement adaptés aux pratiques artistiques ibériques, offrant ainsi la possibilité à leurs clients d'acquérir une œuvre hybride, alliant traditions hispanique et flamande.

Ce cas de figure s'observe notamment en la personne de Juan Rodriguez de Fonseca (1451-1524), un riche ecclésiastique au service de Ferdinand d'Aragon qui s'est rendu dans les Pays-Bas à deux reprises à la demande du souverain pour obtenir des informations sur la manière dont se déroulait le mariage de Philippe le Beau et Jeanne de Castille ${ }^{52}$. Lors de ses séjours bruxellois, le prélat semble avoir développé une dévotion particulière au culte des Sept Douleurs de la Vierge. En effet, il est non seulement connu pour son inscription à la confrérie NotreDame des Sept Douleurs de l'église Saint-Géry de Bruxelles, mais aussi pour avoir passé commande à Jan Joest van Kalkaar d'un retable dédié à ce thème ${ }^{53}$. En tant qu'évêque de Palencia, il destinait cette cuvre au maître-autel de la cathédrale de la ville. Elle se trouve encore aujourd'bủ à son emplacement d'origine.

Le Retable des Sept Douleurs de la Vierge de Jan Joest se présente de manière hybride, sous la forme d'un triptyque dont les volets comportent exclusivement du texte et dont le panneau central revêt la forme d'un retable mural ibérique. Il est en effet divisé en trois travées verticales, la partie centrale étant occupée en bas par un panneau de plus grand format. Celui-ci comporte une effigie de la Vierge éplorée, soutenue par saint Jean et adorée par Juan Rodríguez de Fonseca. Les sept autres compartiments illustrent les épisodes douloureux de la vie de Marie. La formule du triptyque aux volets ornés d'inscriptions est typique des productions brugeoise et bruxelloise du XVI" siècle. Le retable de Fonseca allie donc les traditions ibérique et flamande, ce qui a d'ailleurs conduit Didier Martens à le désigner comme un «triptyque-retablo» témoignant des goûts disparates de son commanditaire ${ }^{54}$. Ce phénomène d'hybridation va même plus loin. En effet, si l'on se concentre uniquement sur le panneau central, construit selon un schéma structurel ibérique,

52 Sur Juan Rodriguez de Fonseca, on consultera notamment A. SAgarRa Gamuzo (éd), Juan Rodriguez de Fonseca: su imagen y su obra, Valladolid, 2005. Voir aussi l'article de Gilles Docquier dans le présent volume.

53 Palencia, cathédrale San Antolín. Sur cette œuvre, voir notamment D. MarTens, Peinture flamande et goût ibérique, op. cit., Bnuxelles, 2010, pp. 220-221 et p. 224 (reproduction p. 221).

54 D. MARTENs, Peinture flamande et goût ibérique, op. cit., p. 224. 
on observe que chaque compartiment pris individuellement apparait comme typiquement flamand, tant au point de vue du style que de la composition. II suffit, pour s'en convaincre, d'observer les panneaux de la Fuite en Égypte ou de la Crucifixion. Si l'on se penche à présent sur le panneau principal, représentant la Vierge soutenue par saint Jean et adorée par Juan Rodríguez de Fonseca. on constate qu'il est, lui aussi, emblématique de ce processus de mélange des tradinions flamande et ibérique : le dévot est représenté sur le même panneau que les saints ; la proximité physique et le contact visuel établi entre eux sont caractéristiques. on l'a vu, des portraits flamands. Par contre, la représentation à échelle légèrement réduite de Fonseca est plus typique des portraits espagnols. Dans le retable de Palencia, Jan Joest mêle ainsi subtilement les deux traditions artistiques st ce, probablement dans le but de répondre aux attentes de son commanditaire et plus largement à celles du public espagnol, tout en conservant les qualités picturales pour lesquelles la peinture flamande était alors réputée. Nous sommes donc ici en présence d'un parfait exemple d'un phénoméne que Didier Martens appelle l'《exotisme flamand mitigé $\aleph^{55}$.

Réalisé par un artiste brugeois anonyme vers 1530 pour l'église paroissiale de Bergara (Pays basque), le Retable de saint Michel est, comme Didier Martens l'a montré, un autre exemple de cet exotisme flamand mitigé relatif au format des retables ${ }^{56}$. Les dévots portraiturés dans cette cuvre n'ont pas pu être identifiés avec certitude, mais il pourrait s'agir de membres de la famille Ozaeta, qui occupe alors une position prépondérante dans la ville basque (fig. 4) ${ }^{57}$. Contrairement au retable de Juan Rodríguez de Fonseca, le Retable de saint Michel est typiquement ibérique d'un point de vue structurel : il s'agit d'un retable mural composé de trois travées verticales divisées en trois registres. Comportant une prédelle, l'ensemble est présenté dans un encadrement gothico-renaissant assez massif et caractéristique des retables muraux espagnols, bien qu'il semble avoir été réalisé en Flandre ${ }^{58}$. La famille de dévots est répartie sur les panneaux inférieurs des deux travées latérales. Les hommes figurent à gauche et les femmes à droite. Le père et la mère apparaissent à l'avant, agenouillés sur leur prie-Dieu et présentés par leurs saints patrons, tandis que leurs douze enfants sont répartis derrière eux. Un vaste paysage flamand s'étend à 1'arrière-plan. Comme dans le retable de Palencia, les différents

55 Voir en particulier l'introduction de l'ouvrage de Didier Martens.

56 Cf. D. MARTENS, Peiniture flamande et goût ibérique, op. cit., pp. 215-217.

57 J. K. STEPPE, Mécénat espagnol et art flamand au XVT siècle, op. cit., pp. 502-503. Les armes peintes sur les prie-Dieu des dévots sont celles de la ville de Bergara. Le retable se trouve aujourd'hui dans la sacristie de l'église San Pedro, mais il ne s'agit pas de son emplacement original. Dans sa notice consacrée à l'ouvre, Jan Karel Steppe mentionne l'existence d'un autel dédié à saint Michel, dont la décoration fut en partie confiée entre 1525 et 1535 au marguillier flamand Juan de Gante. L'auteur considère cependant que le caractère familial du retable de saint Michel empêche d'y voir le retable destiné à cet autel. Il provient selon luì de la chapelle privée de la famille Ozaeta. À nos yeux, cet argument n'est pas valide; 'hypothèse de l'autel dédié à saint Michel et décoré par Juan de Gante nous paraît plus fiable.

58 D. Martens, Peinture flamande et gout iberique, op. cit., p. 217. 


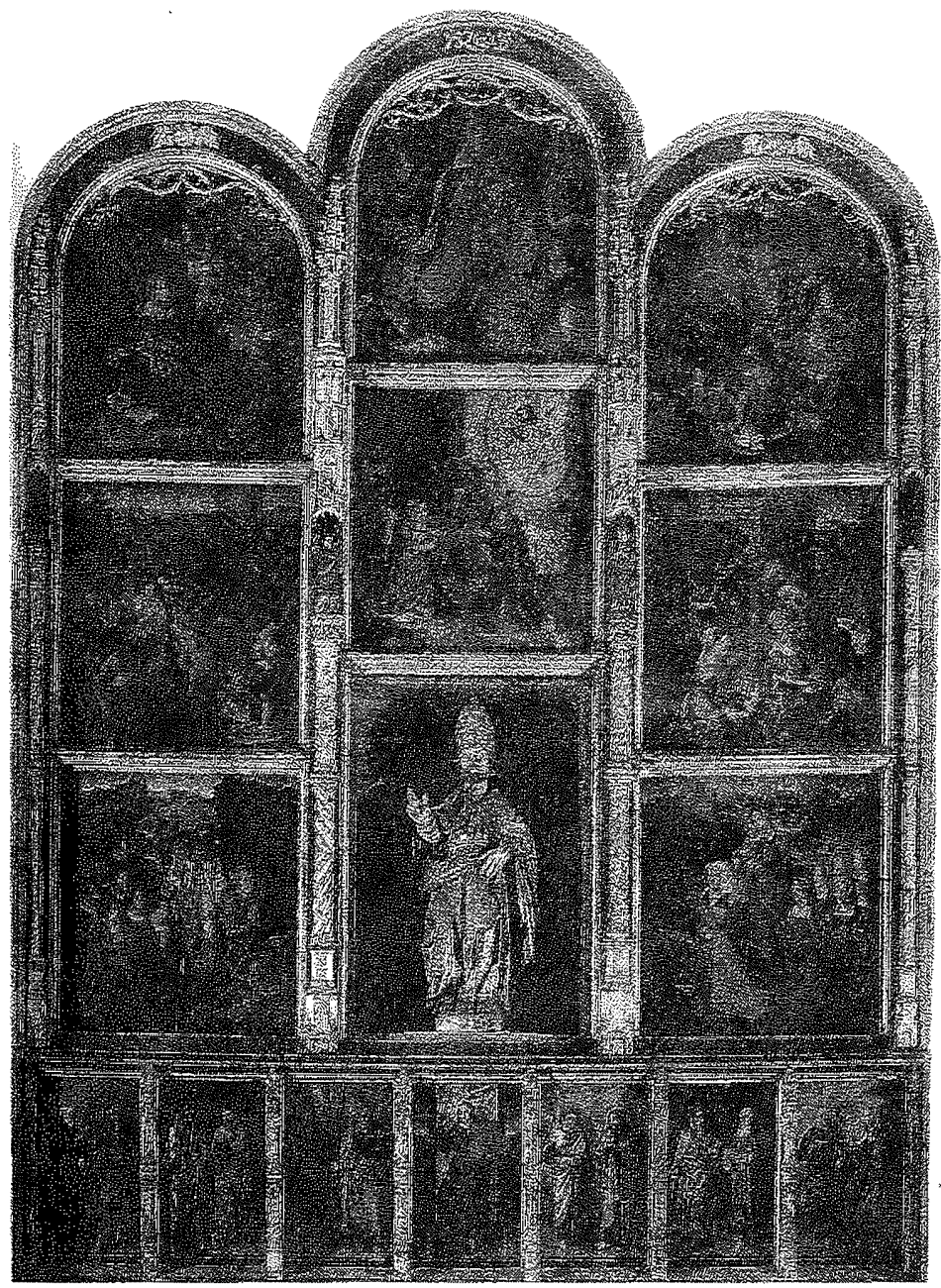

Figure 4

panneaux du retable apparaissent comme typiquement flamands lorsqu'ils sont envisagés individuellement. Cela est particulièrement vrai en ce qui concerne les deux panneaux comportant les portraits et ce, grâce au paysage flamand et à la disposition des effigies. Considéré dans son ensemble, le Retable de saint Michel apparaît toutefois comme une cuvre ibérique.

Le Musée du Prado héberge sept panneaux réalisés par Ambrosius Benson vers 1540 pour le couvent dominicain Santa Cruz la Real de Ségovie. Ils représentent sainte Anne trinitaire, la Rencontre à la porte dorée, la Naissance de la Vierge, la Déposition, la Mise au tombeau, saint Thomas et, enfin, saint Dominique 
accompagné d'un homme en prière ${ }^{59}$. On s'accorde généralement pour affirmer que les cinq premiers tableaux proviennent $d^{s}$ un seul et même retable mural dédié à sainte Anne ${ }^{60}$. Il semble par contre que les deux derniers panneaux, représentant saints Thomas et Dominique, proviennent d'un second retable mural - auquel il faut ajouter une Sainte Catherine de Sienne (collection privée) - qui se trouvait au-dessus de l'autel dédié à la sainte dans l'église conventuelle de Ségovie ${ }^{61}$. Si tel est bien le cas, cet ensemble est ainsi un troisième tómoignage du goût porté par les commanditaires espagnols pour des cuvres comportant des portraits dévotionnels « à la flamande », mais réalisées selon la mode iberique.

Il nous faut mentionner ici une cuvre quelque peu atypique. Il s"agit d"vn Triptyque de la Crucifixion avec une famille en prière réalisé par un artistue bruxellois vers 1500 pour une chapelle de l'église San Pedro de Zumaya, sur ta côte basque (fig. 5) ${ }^{62}$. L'ouvre se trouve encore aujourd'hui à son emplacenent d'origine et relève d'une commande de la fanille Elorriaga, comme l'at aste le blason peint sur le panneau central. Le recours a formule du triptyque - la plus traditionnelle aux Pays-Bas - pourrait avoir de quol étonner dans le cas de ce retable destiné à la péninsule ibérique. Le Triptyque de la Crucifixion se disingue toutefois des triptyques flamands standards par sa structure narrative atypique. En effet, il comporte des volets à compartiments doubles, c'est-à-dire divisés en deux registres et comprenant donc deux scènes religieuses ${ }^{63}$. Comme l'a montré Didier Martens, la plupart des triptyques à compartiments doubles ont été commandés par des Ibériques; il s'agit donc là d'un autre phénomène d'hybridation entre tradicions flamande et espagnole ${ }^{64}$. La présence de tels volets explique que les dévots figurent

59 Madrid, Museo del Prado, $\mathrm{n}^{\circ}$ inv. 1303-1304-1927-1928-1929-1933-1935. Sur ces panneanx, voir G. MARLIER, Ambrosius Benson et la peinture à Bruges au temps de Charles Qumit, Damme, $1957, \mathrm{n}^{\circ} 1$.

60 Voir en dernier lieu D. MARTENs, Peinfure flamande et goût ibérique, op. cit., pp.149-154.

61 L'hypothèse selon laquelle les sept panneaux proviennent du même ensemble a été proposée par Georges Marlier. Depuis lors, Maria Luisi Gómez Nebreda, Pilar Silva Maroto et Didier Martens ont rejeté cette hypothèse et suggèrent l'exîstence du second ensemble. Veìr D. MARTENS, Peinture flamande et goût ibérique, op. cit., p. 154.

62 Sur cette ceuvre, on consultera D. MARTENs, Une oeuvre hispano-flamande du groupe tau Feuillage brodé : le triptyque de Zumaia (Gipuzkoa), dans Le Maitre au feuillage brodé. Démarches d'artistes et méthodes d'attribution d'aunres à un peintre anonyme des anciens Pays-Bas du XV siècle. Colloque organisé par le Palais des Beaux-Arts de Lille les 23 et 24 juin 2005, F. GOMBERT et D. MARTENs (éd.), Lille, 2007, pp. 69-86.

63 Il s'agit, à gauche, du Christ au jardin de Gethsémani et du Portement de croix et, à droite, de la Déploration et de la Résurrection.

64 D. MARTENS, Peinture flamande et goût ibérique, op. cit., pp. 25-97. Les commanditaires de triptyques à compartiments doubles apparaissent comme des personnes ayant désiré concilier leur goût ibérique - à savoir une certaine profusion d'images, typique des retables muraux espagnols - avec les traditions artistiques flamandes. Les volets à compartiments doubles autorisent la présence d'un plus grand nombre de scènes religieuses et permettent ainsi d'associer les deux traditions artistiques en une seule cuvre. Cette formule compositionnelle témoigne ainsi de l'intérêt porté par les Espagnols et les Portugais pour cet « exotisme flamand mitigé ». 


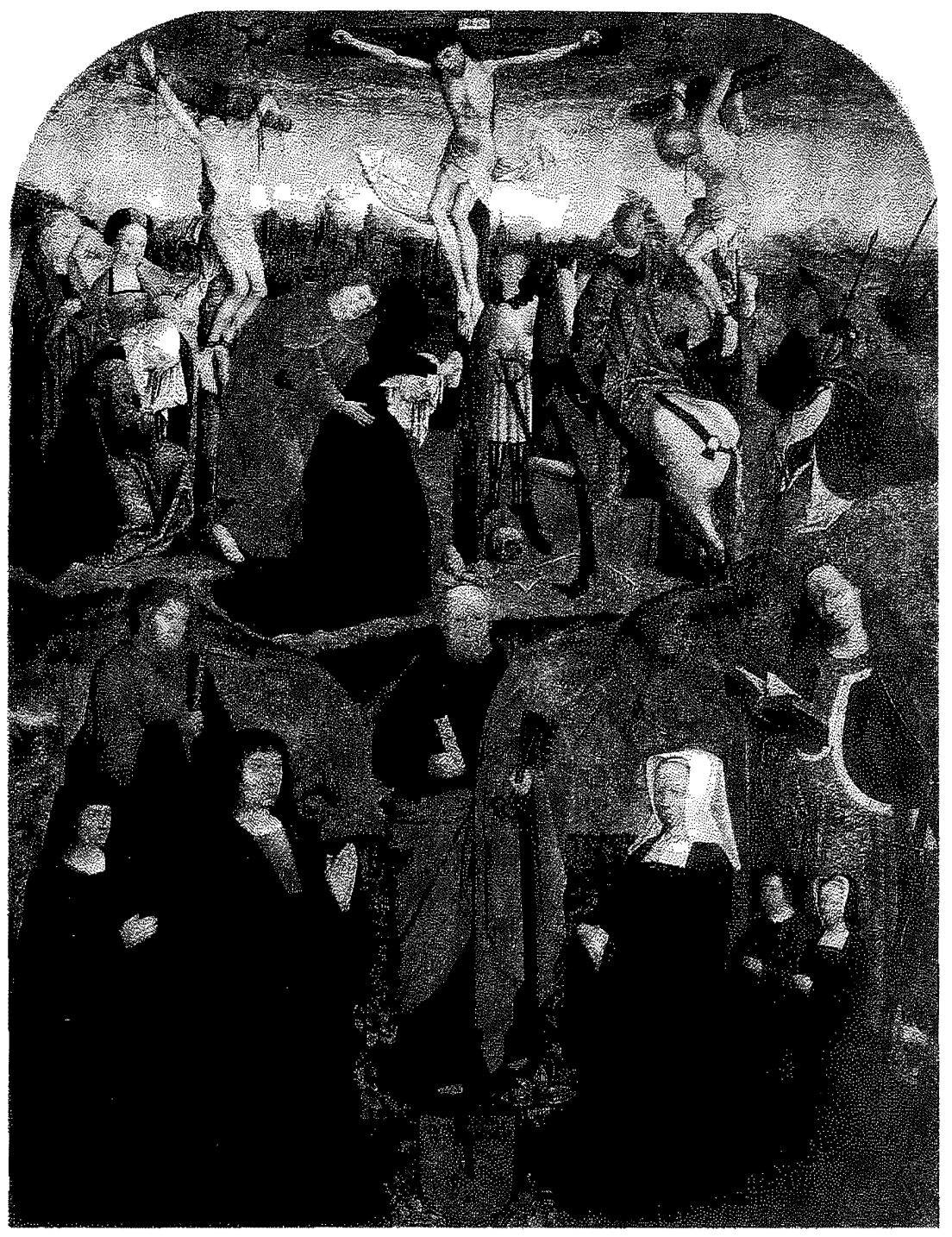

Figure 5 
sur le panneau central, et non sur les panneaux latéraux comme c'est plus souvent le $\operatorname{cas}^{65}$. Autre fait étonnant, les membres de la famille Elorriaga n'adressent pas leurs prières au Christ, mais à saint Pierre, représenté sous la Crucifixion. Cette juxtaposition d'une figure hiératique et d'une scène narrative sur le panneau central évoque la structure narrative des retables muraux espagnols et des retables flamands de format ibérique comme celui de Fonseca et des Ozaeta. De même que les volets, le panneau central du triptyque contribue aussi au caractère hybride de l'ensemble.

Les retables à portraits dévotionnels évoqués jusqu'à présent témoignent du goût de nombreux Espagnols pour des ouvres flamandes s'adaptant partiellement aux traditions ibériques. Tous n'ont cependant pas désiré des ceuvres de ce type. Ainsi, plusieurs artistes flamands se sont vus confier par des Espagnols la réalisation d'cuvres à portraits dévotionnels typiquement flamandes et pourtant destinées à la péninsule ibérique. Parmi ces peintres figure Ambrosius Benson, dont il a déjà été question précédemment. Au long de sa carrière à Bruges, Benson a établi des contacts, tant personnels que commerciaux, avec plusieurs Espagnols établis dans la ville. Ainsi, plusieurs documents d'archives nous renseignent sur les relations qu'il entretient avec le marchand Lucas de Castro - à qui il achète notamment une maison en échange de huit tableaux - ainsi qu'avec un certain Sancho de Santander ${ }^{66}$. Aujourd'hui encore, nombre d'œuvres produites par le peintre et son atelier figurent dans le patrimoine des églises espagnoles. Comme le suggèrent les archives, Sancho de Santander a dû jouer un rôle non négligeable dans l'exportation de ces tableaux vers la péninsule.

Parmi eux, on retrouve de petits tableaux de dévotion privée, mais aussi des retables d'autel, dont deux comportent des portraits dévotionnels. Il s'agit du Triptyque de la Lamentation de l'église paroissiale de Nájera ${ }^{67}$ (fig. 6) et du Triptyque de l'Assomption provenant de l'ermitage de Santa Maria, près de Navarrete ${ }^{68}$, dont on ne connaît malheureusement pas l'identité précise des personnes portraiturées. Dans les deux cas, Benson a opté pour un triptyque chantourné comportant une scène religieuse sur le panneau central et les portraits de dévots accompagnés de leurs saints patrons sur les volets. Le peintre a ainsi opté pour la formule de retable à portraits la plus caractéristique de la production picturale flamande.

65 On remarquera toutefois que la figuration des dévots sur le panneau central du triptyque n'est pas un phénomène rare, même lorsqu'il s'agit d'une famille. Dans le cadre de notre thèse, nous avons recensé dix-huit triptyques de ce type. Parmi eux, huit comporte des portraits de couples ou de familles.

66 Sur ces relations, voir G. MarLier, Ambrosius Benson, op. cit, pp. 23-25 et 34-39.

67 Voir la rotice du catalogue de la vente Sotheby's à Londres du 9 juillet 2008 , lot $n^{\circ} 9$.

68 Navarrete, église Santa Maria. Cf. F. Fernandez PARDO, Las tablas flamencas en la Ruta Jacobea, Logroño, 1999, pp. 291-292 (reproduction p. 291). 



Figure 6

Quelques décennies auparavant, Hans Memling a également produit un triptyque flamand traditionnel et ce, pour un membre de la famille Rojas. On n'en conserve malheureusement plus que le volet gauche, représentant cet Espagnol en prière dans une loggia ${ }^{69}$. Dans le paysage de l'arrière-plan, on reconnaît Joseph d'Arimathie devant le tombeau vide du Christ, ce qui suggère que le panneau central du triptyque représentait la Résurrection, ou plus vraisemblablement te Christ ressuscité trônant. En effet, la présence du dévot dans une loggia - qui se prolongeait certainement sur les autres panneaux de l'wuvre comme c'est généralement le cas - le suggère plus volontiers. Quoi qu'il en soit, l'effigie de Rojas et l'œuvre dans son ensemble sont non seulement caractéristiques de la peinture des Pays-Bas, mais aussi plus précisément de celle de Memling. En effet, ce dernier s'est très souvent plu à figurer ses commanditaires en prière dans des loggias, à l'instar de son Triptyque de John Donne of Kidwelly ${ }^{70}$ ou de son Triptyque de la Vierge à l'Enfant de Vienne ${ }^{71}$.

69 Londres, vente Sotheby's du 10 juillet 2002, lot $\mathrm{n}^{\circ} 8$. La plupart des historiens de $1^{\prime}$ art s'accordent pour identifier cet homme à Francisco de Rojas (1446-1523), ambassadeur des rois catholiques auprès de la cour bourguignonne. Voir la notice du catalogue de vente, ainsi que D. DE Vos, Hans Memling, op. cit., $n^{\circ} 2$ et B. G. LANE, Hans Memling. Master Painter in Fiffeenth Century Bruges, op. cit., pp. 322-323 (reproduction p. 323).

70 Londres, National Gallery, $\mathrm{n}^{\circ}$ inv. NG 6275. Cf. B. G. LANE, Hans Memling. Master Painter, op. cit., pp. 282-284 (reproduction pl. 14).

71 Vienne, Kunsthistorisches Museum, $\mathbf{n}^{\circ}$ inv. 939-943-994. Cf. idem, pp. 316-318 (reproduction p. 317). 
Tout comme le triptyque Rojas, le Triptyque de l'Adoration des mages du Maître de la Légende de sainte Marie-Madeleine conservé à Oviedo est un témoignage du goût de certains Ibériques pour les retables « à la flamande $»(\text { fig. } 7)^{72}$. Le triptyqu a été commandé par Alvaro de Carreño et son épouse María Gonzáles de Quirós pour leur chapelle privée dans l'église San Tirso d'Oviedo, comme le stipule une inscription située sur le cadre ${ }^{73}$. Les époux apparaissent à mi-corps sur les volets en présence de leurs saints patrons, Jacques et Pierre, tandis que le panneau central représente l'Adoration des mages, elle aussi à mi-corps. Les peintres flamands ont fréquemment recours à ce type de cadrage, mais plutôt pour les petits tableaux de dévotion destinés à un usage privé, qu'ils écoulent sur le marché libre ou qu'ils destinent à l'exportation, notamment dans la péninsule ibérique. Ils l'emploient par contre très rarement pour les retables, préférant dans ce cas opter pour le cadrage en pied $^{74}$. On est donc en droit de se demander ce qui a poussé le Maître de la Légends: de Marie-Madeleine et Alvaro de Carreño à choisir cette formule. La raison de ce choix inhabituel semble résider dans le désir du commanditaire de posséder une cuvre flamande clairement identifiable en tant que telle, contrairement à Juan Rodríguez de Fonseca, aux Elorriaga et aux Ozaetz. La formule du triptyque ei le cadrage à mi-corps - tous deux rares dans la peinture ibérique, mais pas flamande contribuent en effet à ce souhait.

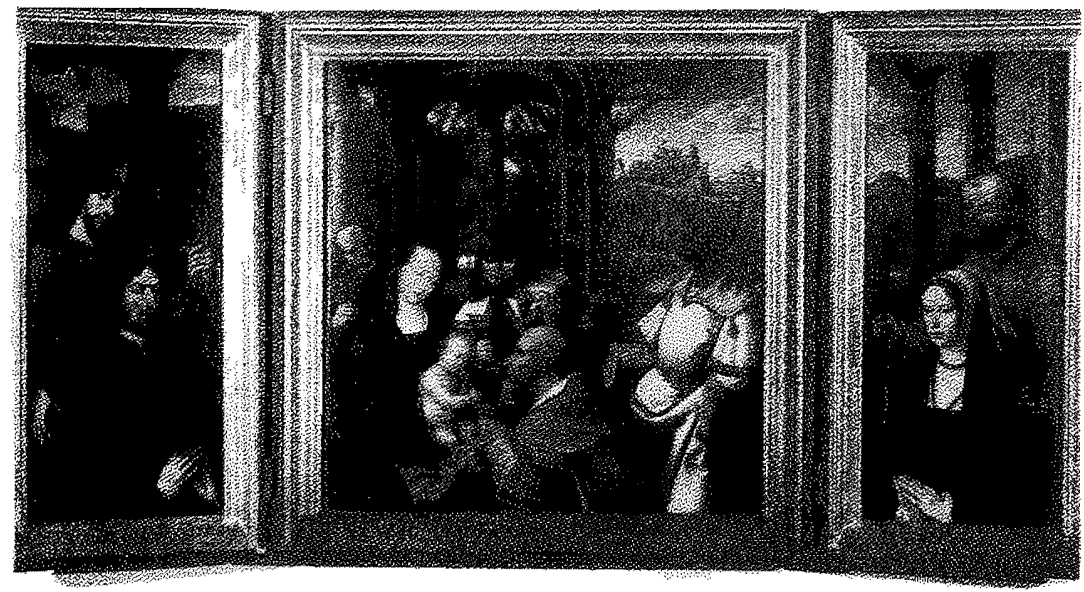

Figure 7

72 Cf. E. Bermeso, Museo de Bellas Artes de Asturias. Obras selectas, Oviedo, 1980, n\% E. Bermero, Algunas obras inéditas del Maestro El Maestro de la Leyenda de la Magdalena, dans Liber amiconum Herman Liebaers, éd. F. VAN WIJnGaERden, J.-M. DuvosQURL, J. Mél ARde L. VIAENE-Awoutzers, Bruxelles, 1984, pp. 395-403.

73 ESTA OBRA MANDO FACER EL HONRADO SEÑOR DON ALBARO DE CARREÑO EN $B R V S E^{i a s} E N E L$ MES.... On remarquera avec intérêt que le lieu de production de l'œưre Bruxelles - est explicitement mentionné dans l'inscription.

74 On recense, bien sûr, quelques exceptions, parmi lesquelles le Triptyque Haneton de Bemard van Orley (Bruxelles, Musées royaux des Beaux-Arts de Belgique, $n^{\circ}$ inv. 358). 
Le Musée dù Prado conserve un tableau attribué à un suiveur de Bemard van Orley représentant Hernán Gómez Dávila, un noble espagnol, en prière devant la Vierge à l'Enfant ${ }^{75}$. Selon une formule compositionnelle bien ctablia dans la peinture flamande de l'époque, la Vierge et le dévot se font face : Marie est assise sur un trône surmonté d'un dais à gauche, tandis que Hernán Gómęz Dávila est agenouillé à droite. Le seigneur de Villatoro a opté, tout comme Jean de Sedano, pour un panneau indépendant comportant son effigie selon une tradition ty piquement flamande. Dans ce cas précis toutefois, l'adaptation à la mode flamande est plus étonnante, d'une part car l'ceuvre est destinée à la chapelle funéraire de la famille Dávila au monastère Saint-François d'Ávila, et d'autre part car le commanditatire n'est probablement pas aussi familier de l'art flamand que Sedano. Quoi qu'il en soit, l'œuvre témoigne particulièrement bien de la volonté des nobles espagnools d'imiter leurs souverains en commandant des ceurvres flamandes.

Excepté le tableau évoqué à l'instant, toutes les cuvres qui viennent d'être analysées sont des retables destinés aux chapelles privées de leurs commanditaires, en terre flamande pour certains et dans la péninsule pour les autres. Parmi les cuvres envisagées dans cette étude, certaines ne sont pas des retables, mais des tableaux de dévotion privée, plus précisément des diptyques à portrait dévotionnel. Comme les triptyques analysés plus haut, ces cuvres trahissent la volonté de leurs propriétaires de posséder un objet typiquement flamand.

Associant l'effigie de la Vierge à l'Enfant et le portrait d'un homme en priere, les diptyques à portrait dévotionnel relèvent d'une formule picturale tout à fait particulière ayant connu un certain succès dans les anciens Pays-Bas entre 1450 et 1530 environ $^{76}$. On conserve deux diptyques entiers et le fragment d'un troisiòme commandés par des Espagnols ${ }^{77}$. Le premier d'entre eux est une diptyque réalisé

75 Madrid, Museo del Prado, $\mathrm{n}^{\circ}$ inv. P1934. Issu d'une des familles les plus notables de Castille et seigneur de Villatoro, Hernán Gómez Dávila est le fils de Gonzalo Dávila, qui tait précepteur du prince Jean. Cf. S. CABALLERo EsCAmLLA, Nouvelles recherches sur la vierge à l'Enfant de Petrus Christus conservée au Musée du Prado, dans Annales de la Société royale d'archéologie de Bruxelles, 69, 2008, pp. 47-48.

76 Diffusé dans les Pays-Bas grâce aux ducs de Bourgogne - en particulier grâce à Philippe le Bon -, le diptyque à portrait dévotionnel a connu trois phases de succès : de 1450 à $1460 \mathrm{au}$ sein de l'aristocratie bourguignonne, durant les deux dernières décennies du XV' siècle at le début du XVI ${ }^{e}$ siècle dans la bourgeoisie brugeoise et enfin dans l'entourage de Marguerite d'Autriche. Sur ce sujet, nous nous permettons de renvoyer à : I. Falque, "Ung petit lableau fermant a deux fuillexz ». Notes sur l'évolution formelle et les voies de diffusion du digtique à portrait dévotionnel dans les anciens Pays-Bas aux XV et XVT' siècles, dans Le Moyen Áge (sous presse).

77 Le troisième témoin est un Portrait d'un jeume homme de la famille Fonseca attribué au Maître des Portraits princiers (Rotterdam, Museum Boijmans van Beuningen, $\mathbf{n}^{\circ}$ inv. 2311). $N^{\prime}$ étant pas conservé dans son intégralité, il n'est pas envisagé davantage ici. Sur cette ceavre, voir Van Eyck to Bruegel. Dutch and Flemish Painting in the Collection of the Museum Boijmans van Beunigen, Rotterdam, 1994, $\mathrm{n}^{\circ} 10$. 
vers 1515-1518 par Michel Sittow pour Diego de Guevara ${ }^{78}$. Il représente, à gauche, la Vierge à l'Enfant et, à droite, 1'Espagnol en prière, les deux panneaux śtant unifiés par le même fond neutre et par une table placée à l'avant-plan des deux panneaux. Comme le signale Antoine de Lusy dans son journal, Diego de Guévara est de nassion d'Espaigne, mais illa esté de toute jeunesse norry en court de par dess $a^{79}$. Par la suite, il occupe plusieurs postes importants au sein de l'administration bourguignonne ${ }^{80}$. Grand amateur d'art, l'Espagnol est également connu pour avoir offert le Portrait des époux Arnolfini de Jan van Eyck à Marguerite d'Autriche. De toute évidence, il s'agit d'un connaisseur qui partage avec la régente son goût pour la peinture nordique. Il $\mathrm{n}^{\prime} \mathrm{y}$ a donc rien de très étonnant à ce qu'il ait commandé un diptyque de dévotion à la Vierge comportant son effigie, tout comme Marguerite d'Autriche 1'avait fait quelques années plus tôt ${ }^{81}$.

Diego de Guevara est un Espagnol qui a passé la majeure partie de sa vie dans les Pays-Bas. Lorsqu'il passe la commande de son diptyque à Sittow, il est donc bien acclimaté à la culture visuelle de cette région. Il ne doit pas exactement en aller de même pour le deuxième commanditaire espagnol, Juan Zúñiga y Avellaneda, qui a chargé Jan Gossart de réaliser un diptyque à son effigie (fig. 8) ${ }^{82}$. En tant que clerc au service de Mencia de Mendoza, il accompagne cette dernière aux PaysBas en 1530 et y reste jusqu'au décès de son époux Henri II de Nassau en 1538. C'est selon toute vraisemblance à l'occasion de ce séjour que, désireux de ramener un souvenir pieux de son voyage dans le Nord, il commande le diptyque au peintre anversois. Tout comme le Diptyque de Diego de Guevara, l'cuvre se présente de manière très traditionnelle : cadrés à mi-corps, la Vierge à l'Enfant et Juan Zúuñiga

78 Berlin, Gemäldegalerie, $\mathrm{n}^{\circ}$ inv. 1722 (volet gauche) et Washington, National Gallery of Art, $\mathrm{n}^{\circ}$ inv. 1937.1.46 (volet droit). Cf. Prayers and Portraits. Unfolding the Netherlandish Diptych, éd. J. O. Hand, C. A. MetzGer et. R. Spronk, New Haven et Londres, 2006, n 34 (avec reproduction).

79 ANTone DE Lusy, Le journal d'un bourgeois de Mons. 1505-1536, éd. A. LouAnt, Bruxelles, 1969, p. 160.

80 En 1501, il est nommé maître d'hôtel de Philippe le Beau. Il accompagne ensuite ce dernier et son épouse Jeanne de Castille en Espagne en 1506, en tant que premier maitre d'hôtel de Jeanne. Il mènera par la suite diverses missions diplomatiques en Espagne et en Angleterre avant d'être nommé receveur des revenus royaux pour la Castille en 1517. La même année. il est nommé clovaria (directeur) de l'ordre de Calatrava, dont il porte l'insigne sur le diptyque. Il meurt vers 1520-1521. Cf. R. FAGE⿺, De hispano-vlaamse wereld. De contacten tussen Spanjaarden en Nederlanders. 1496-1555, Bnuxelles, 1996, pp. 326-327 et H. Coous, Mannen met macht. Edellieden en de moderne staat in de Bourgondisch-Habsburgse Landen $1147 \mathrm{j}$ 1530), Zutphen, 2001, pp. 225-226. Voir également l'article de Gilles Docquier dans le présent volume.

81 Nous faisons référence ici au Diptyque de Marguerite d'Autriche réalisé par le Maitre de 1499 entre 1501 et 1504 (Anvers, Koninklijk Museum voor Schone Kunsten, $n^{\circ}$ inv. 1973-A). Le diptyque de Bernard van Orley comportant son effigie est, quant à lui, daté des environs de 1518. L'original est perdu, mais l'on conserve plusieurs copies, dont une à la National Gallery of Canada à Ottawa ( $\mathrm{n}^{\circ}$ inv. 28531).

82 Voir en dernier lieu M. W. Aussworth (éd.), Man, Myth, and Sensual Pleasures. Jan Gossart's Renaissance, New Haven, Londres et New York, 2010, pp. 298-299. 



Figure 8

se détachent d'un fond sombre. Tout comme dans le diptyque de Guevara, le recours à un décor neutre - et non à un intérieur domestique ou un paysage comme c'est généralement le cas depuis les années 1480 dans la production de diptyques flamands - est sans conteste une « référence-hommage » à la prestigieuse tradition du diptyque dévotionnel aristocratique initiée par Rogier van der Weyden à la cour de Bourgogne.

Pour clore ce panorama des tableaux flamands comportant des portraits d'Espagnols, il reste à envisager une dernière cuvre, atypique à plusieurs égards. Il s'agit d'un tableau votif conservé à l'église paroissiale de Zumaya ${ }^{83}$. Réalisé en 1475 par un artiste anonyme, il se divise en deux registres. En haut, on trouve une scène d'adoration traditionnelle. La Vierge siège sur un imposant trône au centre d'un jardin clos par un haut mur de briques. Elle est entourée, à droite, par sainte Catherine et, à gauche, par saint Pierre et un homme en prière. Le registre inférieur constitue l'élément inhabituel de l'œuvre. Il représente en effet une bataille navale entre quatre bateaux. L'inscription courant sur le chanfrein inférieur nous apprend que l'cuvre est un tableau votif réalisé en 1475 à la demande du marinier et militaire Juan Martinez de Mendaro, lequel souhaitait célébrer sa victoire contre la flotte portugaise lors de la bataille de Gibraltar par le placement de cette image dans sa chapelle de l'église San Pedro de Zumaya ${ }^{84}$. L'adjonction d'un panneau représentant un événement historique à une scène votive traditionnelle est - à notre

83 Zumaya, église paroissiale. Cf. F. Fernandez Pardo, Las tablas flamencas en la Ruta Jacobea, op. cit., p. 105 (avec reproduction).

84 Cf. M. A. ArRaZAda, Renacimiento en Guipúzcoa, t. II, San Sebastián, 1988, p. 334. 
connaissance - une formule inédite, tant dans la peinture flamande que dans la peinture espagnole. Le tableau de Juan Martinez de Mendaro montre ainsi que les commanditaires espagnols peuvent aussi sortir complètement des sentiers battus.

En guise de conclusion à cette présentation des peintures flamandes comportant des portraits d'Espagnols en prière, un constat s'impose : ces commanditaires ont fait preuve d'une certaine diversité. Plusieurs d'entre eux se sont montrés parfaitement acclimatés à la culture visuelle et aux traditions artistiques flamandes, au point d'acquérir une œuvre typique de cette région. D'autres, par contre, ont fait preuve d'un peu plus de "frilosité» - ou se sont du moins montrés plus attachés à leurs traditions régionales espagnoles - puisqu'ils ont opté pour des auvres hybrides, alliant traditions flamande et ibérique. Toutefois, il importe de souligner que dans ce dernier cas, les traits les plus caractéristiques de l'art du portrait dévotionnel dans les anciens Pays-Bas sont respectés. Cela témoigne, si besoin était, de la popularité dont a joui la peinture flamande et du succès rencontré par le portrait « à la flamande » dans la péninsule ibérique. 


\section{Addendum}

Le 6 juillet 2011, la maison Christie's a présenté lors de la vente Old Master \& British Paintings $\left(\mathrm{n}^{\circ}\right.$ 7981) un triptyque attribué au Maitre de la Légende de sainte Marie-Madeleine représentant, sur le panneau central, la Vierge à l'Enfant accompagnée de saint François et, sur les volets, les effigies d'un couple en prière accompagné de ses saints patrons (lot $\mathrm{n}^{\circ} 110$ ). Cette œuvre, déjà mentionnée par Elisa Bermejo en 1984, se trouvait jusqu'à présent dans une collection particulière de Barcelone. Son passage chez Christie's a permis d'identifier les blasons peints sur le revers des volets comme étant ceux de la famille Canizares, provenant d'Asturies. Les personnes portraiturées sur cette œuvre sont donc espagnoles et le triptyque doit être ajouté aux cuvres étudiées dans le présent article.

Tout comme Alvaro Carreño - qui fit d'ailleurs aussi appel au Maître de la Légende de sainte Marie-Madeleine pour son triptyque -, le couple Canizares a opté pour un triptyque cadré à mi-corps. Ce dernier confirme ainsi la tendance de certains Ibériques à commander des cuvres typiquement flamandes comportant leurs portraits à des artistes actifs dans les anciens Pays-Bas. 